\title{
Partial and complete ruptures of the Achilles tendon and local corticosteroid injections
}

\author{
F. Mahler MD, Dip Sports Med and D. Fritschy MD
}

\section{Introduction}

Hench and co-workers first reported the spectacular effects of a hormone of the adrenal cortex $(17-\mathrm{OH}$ dehydrocorticosterone on the rheumatoid joint ${ }^{1}$. This won them the Nobel prize for medicine in 1950.

Unfortunately, the side effects of intramuscular injections of cortisone were important and it was not until 1951 that Hollander et al. elucidated how steroids could be used locally with reduction of systemic side effects ${ }^{2}$. They showed that hydrocortisone injected locally was much more effective than cortisone in suppressing synovial inflammation, confirming Mason's hypothesis that hydrocortisone rather than cortisone was the principal corticoid with anti-inflammatory activity at tissue level ${ }^{3}$.

This was the beginning of a new era in the treatment of a wide variety of inflammatory pathologies. After an initial period of euphoria due to the spectacular effects of hydrocortisone on most inflammatory conditions came the evidence that steroids injected locally also had adverse effects.

Chandler and Wright reported ten cases of rapidly progressive degenerative arthritis following intraarticular hydrocortisone injections ${ }^{4}$. Later, Mankin and Conger showed diminished synthesis of articular cartilage in the knees of rabbits treated with intra-articular steroids ${ }^{5}$. These initial reports were followed by many others condemning steroids, holding them responsible for conditions such as secondary osteoporosis, aseptic necrosis and tendon ruptures. Since these initial reports, there has been conflicting evidence as to the role of corticosteroids in these pathologies, especially in tendon ruptures.

At present there is still controversy as to whether or not corticosteroids should be held responsible when a tendon rupture occurs following local steroid treatment. One of the commonest sites for tendon rupture is the Achilles tendon. The Achilles tendon (TA) has not been spared the increase in overuse

Address for correspondence: Dr Finn Mahler, 12 Chemin Pont Ceard, 1290 Versoix, Switzerland

This review is based on the author's dissertation for the diploma course in sports medicine of the London Hospital Medical College and is reproduced by permission of J. B. King FRCS, course director.

(C) 1992 Butterworth-Heinemann Ltd 0306-3674/92/010007-08 injuries in recent years and is often the site of acute and chronic inflammation. This has led to the use of local corticosteroid injections into the tendon or to the surrounding paratenon.

Lee was the first to report a case of TA rupture following local corticosteroid injection ${ }^{6}$.

The aim of this dissertation is to analyse critically the experimental and clinical knowledge available concerning corticosteroids and TA ruptures. The first section will relate to the fundamental research concerning the effect of corticosteroids on tendons. The second part will be a literature review relevant to the role of local corticosteroid injections in the pathogenesis of TA ruptures.

\section{Animal and human research related to corticosteroids and tendon ruptures}

\section{Intratendinous injections}

Ferland's study on adult albino rabbit Achilles tendons clearly demonstrates the consequences of an injection of corticosteroid directly into the $\mathrm{TA}^{7}$. The comparison of two groups, one receiving an intratendinous injection of corticosteroid, the other a peritendinous injection, showed that $100 \%$ of the group with the intratendinous injection had localized tendon necrosis at the site of the injection. On the other hand, the peritendinous injection group showed an intact structure in $95 \%$ of cases.

To eliminate the hypothesis that the necrosis could have been secondary to an increased intratendinous pressure, a third group was injected intratendinously with an identical volume of physiological serum $(0.3 \mathrm{ml})$. No necrosis occurred in this group, leaving the corticosteroid solely responsible for the tendon necrosis in the group receiving the intratendinous injection.

A similar study by Balasubramaniam, also on the TA of rabbits and comparing an intratendinous injection of hydrocortisone with an identical volume of saline solution, showed no deleterious effect with the saline injection and invariably a collagen necrosis with the hydrocortisone ${ }^{8}$. Necrosis was seen as soon as $45 \mathrm{~min}$ after injection and after 8 weeks the healing process was still incomplete. Also, the tendons having received the injection into their central part had poorer scar tissue at 8 weeks compared with those where the corticosteroid had been peripherally 
injected. This could be due to a comparatively poorer blood supply to the centre of the tendon.

Unverferth compared the TA tensile strength of two groups of rabbits. ${ }^{9}$. One was injected with corticosteroid and the other with saline solution. The results showed a significant decrease in tensile strength in the group injected with corticosteroids. Microscopic analysis showed disruption of the collagen bundles with deposits of a pale material presumed to be steroids. Unfortunately no histochemical analysis was done, leaving doubt as to the nature of the deposits. The routine staining with haematoxylin and eosin took on an orange, granular appearance, compatible with tendon necrosis. The tendons injected with a saline solution showed insignificant alterations.

Kennedy and Baxter Willis studied the effect of a single injection of $\beta$-methasone into the TA of rabbits and showed a decrease in tensile strength of $35 \%$ after $48 \mathrm{~h}$ and at 7 days ${ }^{10}$. After 2 weeks, no difference was found with controls. In their conclusion, they proposed a period of at least 2 weeks' abstinence from vigorous exercise after a local injection of corticosteroids.

Phelps, in his study on the tensile strength of the patellar tendon of rabbits after multiple injections of methylprednisolone, found no alterations in the mechanical properties of the tendons injected with corticosteroids ${ }^{11}$. He concluded that tendon rupture in athletes could stem from some underlying pathological process and not to a destructive process initiated by the steroid injection.

One of the main problems with animal research is its extrapolation to man. Noyes et al. were able to get closer to the human situation with their study on the anterior cruciate ligament of 110 Rhesus monkeys ${ }^{12}$. They compared tensile strength and histological modifications at 6,15 and 52 weeks between two groups. One group received a direct intraligamental injection of methylprednisolone, the other an intraarticular injection of the same product. The results showed that a single intraligament injection substantially decreased the tensile strength (between 27 and $39 \%$ ) up to 52 weeks after the injection. Histological examination showed death and absence of fibrocytes. In the group which received an intra-articular injection, there were no significant changes in tensile strength or histology.

\section{Peritendinous injections}

Local corticosteroid injections around the tendon are more controversial. Authors such as Goldie deny that corticosteroids should be held responsible for tendon ruptures following local injections ${ }^{13}$. Initial research was designed to find out if steroids could reduce adhesions after tendon surgery.

Carstam showed that the tensile strength of dog Achilles tendon was not altered by the presence of corticosteroids ${ }^{14}$. Gonzalez investigated the local effect of hydrocortisone on tendons repaired within the flexor tunnels ${ }^{15}$. He showed that the functional results and the tensile strength of the tendons bathed in hydrocortisone were no better than those repaired without the drug.
In a more recent study, Vogel showed a surprising increase in the tensile strength of tendons after corticosteroid administration ${ }^{16}$. However, the repetition of injections progressively weakened the tendons, suggesting a relationship between cumulative dose and effect.

The only study showing a decrease in the tensile strength of tendons after corticotherapy is the work by Wrenn and co-workers ${ }^{17}$. They showed that the daily administration to dogs of $10 \mathrm{mg} \mathrm{kg}^{-1}$ bodyweight of cortisone inhibited excessive formation of peritendinous fibrous tissue. On the other hand, the breaking point of the sutured tendons treated with cortisone was consistently lower than in the control group $(40 \%)$. It should be noted that the doses of cortisone used far exceed the relative doses currently used in humans. Furthermore, the relevance of these intramuscular injections remains uncertain.

In the light of these studies tending to show the absence of any deleterious effect of peritendinous injections, how can one explain the reigning controversy as to whether or not peritendinous injections enfeeble the tendon.

Different explanations are possible. First, there is the extrapolation of animal research to humans. Second, all the studies were carried out on tendons without any underlying pathology which is not the case when corticosteroids are used in humans. The defenders of corticosteroids can always claim that the underlying pathological processes of the tendon for which the corticosteroid was used is responsible for rupture. Others remain persuaded that corticosteroids have a direct enfeebling effect on the tendon.

A third view claims that corticosteroids, by reducing inflammation and thereby pain, permit the athlete to resume vigorous exercise, hence exposing the already fragile tendon to rupture.

In the light of these studies, it does not seem reasonable to condemn peritendinous injections by invoking a direct deleterious effect on the tendon itself.

\section{Partial and complete ruptures of the Achilles tendon}

The first precise description of Achilles tendon pathology came from Ambroise Paré in 1575. He described the serious nature of an injury to this tendon, which invariably had an unfavourable outcome. It was not until the end of the 19th century that surgery was performed to ruptured TAs. In 1882, Maydl described for the first time a rupture in a sporting situation (mountaineering). Later, Albrecht (1924), Pirker (1934) and others reported the lesion in athletes, dancers and tennis players. In 1941, Silverskjöld reported seven cases of people with acute ruptures of the TA, all of whom were participating in a sporting activity ${ }^{18}$.

\section{Incidence}

The incidence of TA ruptures is often quoted as rare. In 1969 Goldman et al. retraced 33 cases of presumed or proven complete ruptures in a period of 20 years at the Mayo Clinic ${ }^{19}$. 
In 1954, Christensen found only 57 cases of TA ruptures in the 70000 patients treated in the orthopaedic department of Aarhus Hospital in Denmark $^{20}$. More recently, Barfred suggested that there was a substantial increase in the incidence of this lesion ${ }^{21}$. Nillius confirmed this hypothesis by performing a retrospective study on the incidence of TA ruptures in the population of Malmö between 1950 and $1973^{22}$. He showed that the increase in the incidence of ruptures was significantly greater than the increase of the population itself.

However, in the same period, there happened to be a remarkable increase in the number of people participating in sports, which was characterized by an increase in the enrolment of members in various sporting associations. In so far as enrolment reflects participation in sporting activities, the increase in the incidence of ruptured TAs was proportional to the increased participation in the sports most commonly causing the injury. As to the incidence of ruptures in non-sporting situations, it had also proportionally increased over time. The author had no satisfactory explanation.

Arner and Lindholm found that the incidence of TA ruptures had doubled in their practice since they had actively sought them ${ }^{18}$. This might explain to some extent Barfred's epidemiological finding of an increase in the incidence of TA ruptures.

Concerning the possible influence of local injections of corticosteroids on the incidence of ruptures, there is to date no longitudinal study.

\section{Diagnosis and pathogenesis}

Acute rupture of the TA is typically seen in a mesomorphic, middle-aged man, participating in an intermittent sporting activity ${ }^{23}$. In Arner and Lindholm's study of 92 cases ( 79 men and 13 women), the average age was 38.5 years and the male to female ratio was $6: 1^{18}$. Male predominance was $10: 1$ in Lea and Smith's study ${ }^{24}$.

There is a preceding history of achillodynia before many TA ruptures. This could be a sign of an underlying pathological process. In Ljungqvist's series of 24 cases of partial ruptures, 16 had a history of achillodynia 25 . To understand the causes of achillodynia better, it is important to get an insight into all the different categories of TA pathology. There exist many classifications of injury-related TA pathology. Williams' is probably the most complete that is presently available $e^{26}$. It recognizes four tendon pathologies with distinct clinical findings: complete rupture; partial rupture; focal degeneration; and tendinitis. A separate category is made for paratenon lesions; peritendinitis (acute or chronic). The last category constitutes mixed lesions.

Not included in Williams' classification but often used by authors, including Williams himself, is the term 'tendinosis'. This term was introduced by Puddu to describe the situation of a grossly degenerative tendon in the absence of alterations to the paratenon ${ }^{27}$.

Williams has tried to differentiate tendinitis from 'tendinosis' by pointing out that the essential element of tendinitis is the characteristic reversibility of the process $^{26}$. On the contrary, 'tendinosis' is a degenerative condition and therefore should be considered as a typically irreversible process. One might suggest that 'tendinosis' is a form of advanced focal degeneration. Going even further, one could imagine that an acute inflammatory process evolves progressively into a chronic degenerative process if the repair mechanisms of the tendon are inadequate, or if the physiopathological cause responsible for the initial inflammation is not stopped.

To support this hypothesis, it is interesting to note that, in Puddu's study, all the ruptures showed macroscopic and microscopic degenerative lesions ${ }^{27}$. Their conclusion was that subcutaneous ruptures of the TA were secondary to the underlying degenerative process, in both symptomatic and asymptomatic patients. To support this 'degenerative theory', one can mention Williams' work on patients operated for achillodynia having resisted all conservative treatment ${ }^{28}$. Invariably, there was macroscopic evidence of repeated attempts to form normal scar tissue which had been hindered by recurrent trauma or by insufficient blood supply for the completion of the healing process.

Fox showed that out of 22 patients presenting TA pathology, ten went on to have complete ruptures ${ }^{29}$. Histology showed diffuse degenerative signs in nine out of ten. It is interesting to note that none of these ten patients had received local steroid injections.

Williams showed that in 12 cases of post mortem material of patients without any history of achillodynia, no modifications of the tendon could be found, suggesting that degenerative changes were not part of a normal ageing process ${ }^{26}$. Ippolito et al. did not come to the same conclusion in their post mortem studies ${ }^{30}$. They did find degenerative changes in some cases, but some of their patients had systemic inflammatory diseases which could have caused the tendon lesions.

Taking into consideration these different studies, is it possible to say that all tendons that rupture have an underlying degenerative process? The answer is no. Jacobs showed that only slightly more than $50 \%$ of TA ruptures had degenerative modifications; the rest were normal ${ }^{31}$. So there is little doubt that an acute rupture can happen in a tendon without any underlying disease. It is for this reason that Barfred proposed a purely mechanical theory to explain the pathogenesis of TA ruptures ${ }^{21}$.

Certain authors suggested that, in ageing athletes, there could be a series of microruptures in the collagen fibres following repetitive stresses beyond the elastic threshold of the tendon ${ }^{29,32}$. This would lead to repair processes but at an insufficient rate compared with the repetitive microtraumas to the tendon. Burry suggested a disparity between the metabolic demands of the tendon and the existing blood supply, leading to an ischaemic degeneration $^{33}$.

Lagergren and Lindholm, in their study on the vascular distribution in the TA, showed that the segment of the tendon with the poorest vascularization was situated $4-5 \mathrm{~cm}$ proximal to the calcaneal insertion and suggested that this contributed to its susceptibility to rupture ${ }^{34}$. 
The mechanical theory was brought forward by Barfred to challenge the degeneration theory ${ }^{21}$. The view that the healthy tendon never ruptures had been supported by histology and by rupture experiments on animals. Barfred's study on the rat TA showed that an external force (maximal electrical stimulation of the sciatic nerve) could rupture the tendon.

His experiments raised interesting observations: rupture incidence was highest after inactivity; rupture risk was increased when oblique force was applied; fatigued muscle predisposes the tendon to rupture. Barfred concluded that experimental rupture of healthy tendon was possible in rats and that it was the consequence of many non-pathological factors that did not necessarily all have to be present at the same time.

Arner and Lindholm distinguished between three indirect mechanisms capable of provoking TA rupture in $\operatorname{man}^{18}$ :

1. Propulsive movement of the weight-bearing forefoot during a simultaneous extension of the knee joint, e.g. sprinting.

2. Unexpected dorsiflexion of the ankle joint, e.g. missing a step.

3. Violent dorsiflexion of a plantar flexed foot, e.g. falling from a height on to the plantar flexed foot.

The pathogenesis of TA rupture is probably multifactorial. The classical story suggests that rupture is the sole consequence of a violent, unusual, purely mechanical indirect force. The less classical story suggests a multifactorial aetiology - degenerative, ischaemic and biomechanical.

Mechanical and degenerative factors may coexist. One could imagine a continuum between the two; on one side a purely mechanical rupture and on the other a low energy rupture in a grossly degenerative tendon. In between, a wide variety of situations where the mechanical energy necessary to produce the rupture corresponds to the level of degeneration of the tendon.

\section{Role of corticosteroids in TA ruptures}

Lee first reported TA rupture in a runner injected with corticosteroids on three previous occasions ${ }^{35}$. He suggested that the corticosteroids could have influenced the final stage of the rupture. In his commentary, he did not exclude the possibility that the corticosteroids could have been injected between the superficial and deeper layers of the tendon and absorbed slowly due to the relatively avascular nature of the tissue. He also suggested that the disruption could have been going on for some time, and that simultaneous repair may have been hindered by the hydrocortisone. He also noted that there was no experimental evidence of a deleterious effect of hydrocortisone on tendon healing.

Controversy still exists 30 years later. The majority of Lee's questions are still unanswered. Despite this uncertainty, most orthopaedic, rheumatology and sports medicine text books condemn local corticosteroid injections around the tendon, holding them responsible for secondary tendon ruptures. As proof of their deleterious effect, studies such as those of Lee are quoted. This is highly questionable considering Lee's own uncertainty. Even more astonishing are certain publications dealing specifically with the matter. For example, Rappaport and Gerster, on local steroid injections in rheumatology, stipulate that tendon ruptures follow abrupt movements in sports people and most commonly involve the Achilles or bicipital tendon ${ }^{36}$. It is also said that they are favoured by local corticosteroid injections. This is supported by the work of d'Anglejan ${ }^{37}$. In this article, the author discusses the deterioration and ruptures caused by corticotherapy, stating that 'these accidents seem to be favoured by local or general cortisone treatment, particularly amongst sportsmen, for whom many cases have been reported in the literature. This is based on five references.

The first reference concerns a 48-year-old patient suffering from rheumatoid arthritis ${ }^{38}$. Following two local injections of corticosteroids for achillodynia, she is victim of an acute TA rupture. No allusion to any sporting activity is to be found in this article. It is suggested by the author that the two injections were probably intratendinous, thereby slowing down the maturation of the fibrous tissue and maybe decreasing the tensile strength. The fact that the patient was suffering from rheumatoid arthritis makes it impossible to evaluate the responsibility of the corticosteroids when one takes into consideration that spontaneous rupture of tendons is an integral part of the features of rheumatoid arthritis.

The second reference concerns a 73-year-old patient with a rapidly destructive arthritis after intra-articular injections of hydrocortisone ${ }^{39}$. There is no allusion to any tendon in this article.

The third reference concerns a 52-year-old patient with a 14-year history of chronic lupus erythematosus $^{40}$. Six months after the initiation of oral triamcinolone the patient developed a bilateral TA rupture. There is no mention of any sporting activity. As to the role of the corticosteroids, again it seems impossible to dissociate it from the underlying inflammatory process.

The fourth reference relates to an experimental study on the rabbit $\mathrm{TA}^{7}$. The last reference describes the cases of an 84-year-old man and a 69-year-old lady, both of whom developed TA ruptures after local corticosteroid injections ${ }^{41}$. There is no allusion to any sporting activity.

There is no objective evidence in any of these references to suggest that TA ruptures are caused by corticosteroids. Furthermore, no mention of any sporting activity is to be found in the references. Unfortunately, this is but one of the many examples of affirmations concerning local corticosteroid injections without supporting evidence. Very little literature dealing specifically with the subject exists, and most is anecdotal.

To the author's knowledge, 19 articles exist which specifically relate to this subject. Eight of these $\mathrm{e}^{35,38,40-45}$ relate to TA ruptures in patients taking oral corticosteroids. All except one were taking the steroids because of a systemic inflammatory disease, making it impossible to differentiate the role of the 
underlying disease processes in the aetiology of the rupture from that of the possible influence of the corticosteroids.

In Lee's case report of a bilateral TA rupture in a 61-year-old man taking prednisone for 4 years, it is interesting to note the commentary of Mayer ${ }^{35}$. He describes the case of a perfectly fit 46-year-old woman who suffered a similar injury though she had never taken steroids.

There is no doubt that spontaneous, indirect, bilateral ruptures of the TA do exist in patients not taking corticosteroids. The only case of a TA rupture among these eight articles, where there is no systemic underlying inflammatory disease process, is that of a 68-year-old man on methylprednisolone because of chronic bronchitis. This is yet another anecdotal case, of little help in the analysis of the role of corticosteroids in TA ruptures.

If corticosteroids really had such an obvious deleterious effect on tendons, one would expect an epidemic of tendon ruptures, when one takes into consideration the many patients taking corticosteroids.

Among the articles concerned specifically with local injections, four, including Lee's study, relate to anecdotal cases of ruptures following one or multiple local injections ${ }^{35}$. Chechick et al. reported the case of a professional football player with Achilles peritendinitis, injected on three occasions with prednisolone $^{46}$. Ten days after the last injection the patient was back playing. He suffered TA rupture in a game. No mention is made of whether the injections were intratendinous or peritendinous.

Kleinmann and Gross reported the cases of three TA ruptures in middle-aged people with chronic Achilles tendinitis, all treated with local corticosteroid injections ${ }^{47}$. In two of the cases, it is specified that the injections were given directly into the tendon.

In their discussion the authors firmly believe the corticosteroids to have played a causative role, because the tendon ruptured within 2-6 weeks of steroid injection and after relatively minor trauma. Furthermore, in their experience rupture of the TA is usually a sudden traumatic event with the patient having no preceding history of chronic pain.

Concerning this last point, there is evidence asserting the contrary. Fox studied 19 cases of TA ruptures surgically repaired ${ }^{29}$. About $50 \%$ of the cases had a history of achillodynia before the rupture. None of the patients had received local corticosteroid injections.

Halpern also reported a 34-year-old man with a TA rupture who had had a 4-year history of achillodynia treated on five occasions with intratendinous corticosteroid injections ${ }^{48}$.

Little can be learnt as to the role of local injections of corticosteroids in TA rupture through these anecdotal reports. The only comment one can make is that the majority of the local injections are described as being intratendinous. This has clearly been shown above to have a directly deleterious effect on the tendon and should be unanimously condemned. Unfortunately, no relevant information as to the role of peritendinous injections can be collected from these studies.
In his study on central degeneration of the TA, Burry refers to the possible role of local injections of corticosteroids $^{33}$. He suggests that an injection in the peritendinous region could provoke an increase of pressure sufficient to interrupt blood flow and cause infarction. This has been shown not to be the case in animal studies. Ferland's comparative study on the rabbit TA failed to show any localized tendon necrosis due to an increase in tissue pressure ${ }^{7}$. Balasubramaniam agreed and showed that the corticosteroids were directly responsible for tendon necrosis ${ }^{8}$.

Skoech evaluated 16 partial TA ruptures, nine of which were explored and repaired ${ }^{49}$. Seven of these 16 tendons had previously received between one and five injections of corticosteroids. During surgery, two of the tendons showed signs of acute rupture, three showed granulated tissue or maturing scar tissue within the area of the defect, and the remaining four tendons showed areas of pearly nodular degeneration within the area of defect, some containing areas of calcification.

Unfortunately the proportion of the nine surgically explored tendons previously injected is not stated. This makes it impossible to analyse the role of the corticosteroids in the pathogenesis of these tendon ruptures.

Denstad and Roaas analysed 58 cases of partial TA ruptures that had undergone surgery ${ }^{50}$. In all, 32 cases $(55 \%)$ had had local corticosteroid injections before the rupture.

Shields et al. evaluated the isokinetic force of the musculotendinous unit in 32 cases having undergone surgery for complete TA rupture ${ }^{51}$. Ten had received previous steroid injections, of whom eight $(80 \%)$ had received their local injection after the episode of complete rupture, which had not been diagnosed. In this series, 18 of the 32 patients did not have the diagnosis established until late.

Apart from showing that the repaired TAs had on average a $16.5 \%$ loss of plantarflexion strength and a $17.5 \%$ loss of plantarflexion power, the authors' results demonstrated that the strength and power ratings of the injured leg were not influenced by cortisone injections. Only two patients out of 32 had received corticosteroid injections before the rupture.

The experimental part of Unverferth's study on the effect of local steroid injections showed that intratendinous steroid injection decreases tensile strength' The clinical part of this study reports the cases of three elite athletes with acute TA rupture following steroid injections. The delays between the last injection and the rupture were respectively 2,4 and 36 weeks. In two of the cases, it is clearly specified that the injections were both intratendinous and peritendinous. No details are given concerning the third case. In his discussion, the author adheres to the hypothesis that local corticosteroid injections mask the symptoms, hence permitting the athlete to return to active competition prematurely.

The author also suggests that local steroid injections diminish tensile strength, especially when the injection enters the tendon itself. This statement presupposes that peritendinous injections also decrease tensile strength. In his conclusion it is proposed that all steroid injections are to be 
abandoned, not only because they give the patient a false sense of security, but also because local injection of steroid in and about the tendon decreases its tensile strength and predisposes it to complete rupture. The numerous studies previously enumerated fully support the author's point of view on intratendinous injections. However, there are no arguments in the study permitting the conclusion, as he suggests, that peritendinous injections also decrease tensile strength.

The last study with a specific insight into local corticosteroid injections is that of Ljungqvist ${ }^{25}$. In the chapter on the aetiology of partial rupture, steroid injections are discussed. The material for the study comprises 24 cases of partial ruptures of which six had been given intratendinous and peritendinous corticosteroid injections before the rupture occurred. Four cases had injections before and after; eight cases had been injected solely after the rupture; six patients were never given injections. Accordingly, 14 patients had not received any corticosteroid injection before the rupture. Among the ten patients injected before the rupture, two had a 1-year interval between the injection and the rupture, making it hard for the author to believe that the injection in these cases could have had any particular significance.

Six other patients out of the ten had been given the injections 2-4 months before the rupture. In four of these six, the local symptoms causing injection were at a different site from that of the rupture. The last two cases had been given their injections 5 and 6 weeks before the rupture and were symptom-free until they sustained the rupture.

Histology was performed on all surgical cases. No difference was shown between the group having received local corticosteroid injections and those without any history of corticosteroid injections.

The author concludes that more than $50 \%$ of the patients had never received injections before the rupture. In the others, it was his opinion that the time relationships, among other factors, argued against the possibility that the corticosteroids would have been of direct aetiological significance. However, the author formulates the hypothesis that the corticosteroids 'might have played a part by relieving the patient of the symptoms attending a small rupture or some other injury of the Achilles tendon, and so allowed increased weight-bearing on the tendon, thus being involved in causing the clinically manifest rupture'.

In conclusion, one can say that in the light of all the existing literature to date, it is still impossible to determine with precision the role of local injections of corticosteroids in the pathogenesis of partial and complete ruptures of the TA.

Nevertheless, certain important points can be postulated. First, on the basis of animal experimentation and clinical observations it is clear that all intratendinous injections should be abandoned. Second, there exists no formal proof of any deleterious effects of peritendinous injections. Last, Ljungqvist's hypothesis stipulating that local corticosteroid injections could mask the symptomatology and therefore expose the tendon to further trauma is plausible ${ }^{25}$.

\section{Conclusion}

Animal research has shown that intratendinous corticosteroid injections result in collagen necrosis, followed by a decrease in tensile strength. On the other hand, the majority of studies dealing with peritendinous corticosteroid injections are unable to show any direct deleterious effect to the tendon.

Despite this difference, all the retrospective clinical studies in humans dealing with corticosteroid injections and TA ruptures never attempt to differentiate between these two fundamentally different types of injections. This having been said, one might ask if it is really so easy to distinguish between an intratendinous and a peritendinous injection. Theoretically, the resistance to the injection of the product in an intratendinous injection is said to be much greater than in a purely peritendinous injection. Practically, the distinction might not be that obvious. Furthermore, in the presence of a tendinosis or a partial rupture, it is most probable that the resistance to the injection is altered, thereby giving a false impression as to the exact location of the injection.

We need to identify separately the effects of corticosteroid injections in each distinct group of TA pathologies. If there is a risk in giving corticosteroid injections, is it the same in treating a pure peritendinitis, compared with a tendinitis or a focal degeneration? This brings up the vast issue that is beyond the scope of this dissertation of the actual indications for corticosteroid injections.

There is probably as big a controversy on this subject as there is on the role of corticosteroid injections in tendon ruptures! Hamilton's point of view is that corticosteroids should never be used near a tendon ${ }^{52}$. Renström thinks that they should only be used in chronic situations and that the injection should be given into the paratenon ${ }^{53}$. In Da Cruz's prospective, randomized, double-blind study to evaluate the role of peritendinous injection of methylprednisolone in the treatment of Achilles peritendinitis, the author concludes that corticosteroids have no role to play in the management of this pathology $y^{54}$. There are as many points of view as authors on this controversial subject, and again it is rare that any distinction is made between the different categories of TA pathology.

Another important fact that has led to the controversy on corticosteroid injections and TA ruptures is improper diagnosis. As cited before, it is astounding to note that in Shields' study on complete ruptures of the TA, $25 \%$ of the patients were given corticosteroid injections after the acute rupture ${ }^{5}$. It is absolutely clear that corticosteroid injections have no place in the management of acute ruptures of the TA, but their use in these situations by ignorance or incompetence has probably contributed to our confusion on the subject.

It is only through the selection of rigorously homogeneous groups that one will be able to accomplish the prospective studies necessary to elucidate the fundamental questions remaining unanswered. With the appearance of new imaging techniques, it should be possible to distinguish between the different categories of TA pathology and 
thereby differentiate between degenerative and inflammatory conditions. These two conditions are often sources of confusion when it comes to the evaluation of the role of corticosteroid injections. This is due to the fact that degenerative pathologies are probably intrinsically a risk factor for ruptures. This intrinsic risk is hard to differentiate from the potential added risk of a local corticosteroid injection.

What sort of studies are necessary for a better comprehension of the subject? First, the difference between a peritendinous and an intratendinous corticosteroid injection should be precisely defined. One could imagine a study comparing the tensile strengths of two groups of animals, one receiving an intratendinous corticosteroid injection, the other a peritendinous injection. The ideal model would be the study of Noyes ${ }^{12}$ on the Rhesus monkey, but instead of using the anterior cruciate ligament, one would use the TA.

Another interesting study would be to see if one can produce a cartilaginous and osseous metaplasia in a tendon merely by injecting it with corticosteroids. This is important because the histological examination of material taken from the site of ruptures often describes this modification without it being possible to know if it is a degenerative process of the tendon itself, or if it is the consequence of the local corticosteroid injection.

As to the studies in man, the ethical restrictions are definitely a limiting factor. This is why one is generally obliged to conduct retrospective studies on non-homogenous groups, which unfortunately makes a precise analysis difficult. This having been said, it is surprising that no longitudinal study on the role of corticosteroid injections in partial and complete ruptures has ever been done. Such a study, comparing the long-term evolution of patients treated with local corticosteroid injections and those having never been injected, should be a priority in the future.

\section{References}

1 Hench PS, Kendall FC. The effect of the adrenal cortex and of pituitary adrenocorticosterone hormone on RA: preliminary report. Proceedings of the Staff Meetings of the Mayo Clinic 1949; 24: 181.

2 Hollander JL, Brown EM, Jessar RA. Hydrocortisone and cortisone injected into arthritic joints. JAMA 1951; 147: 1629-35.

3 Mason HL. Urinary excretion of steroids during administration of ACTH. In: Mote JR, ed. Proceedings of the First Clinical ACTH Conference. Philadelphia, USA: Blakiston, 1950: 168-76.

4 Chandler GN, Wright V. Deleterious effect of intra-articular hydrocortisone. Lancet 1958; ii: 661.

5 Mankin HJ, Conger KA. The acute effects of intra-articular hydrocortisone on articular cartilage in rabbits. J Bone Joint Surg 1966; 48A: 1383.

6 Lee HB. Avulsion and rupture of the tendo calcaneus after injection of hydrocortisone. $\mathrm{Br}$ Med J 1957; ii: 395.

7 Ferland MA, Uhthoff HK. Nécrose localisée due à une injection intratendineuse de gluco-corticoide: étude expérimentale comparative. Union Méd Can 1972; 101: 1768-71.

8 Balasubramaniam P, Prathap K. The effect of injection of hydrocortisone into rabbit calcaneal tendons. J Bone Joint Surg 1972; 54: 729-34.

9 Unverferth LJ, Olix ML. The effect of local steroid injections on tendon. I Sports Med 1973; 1: 31-7.

10 Kennedy JC, Baxter R. The effects of local steroid injections on tendons: a biomechanical and microscopic correlative study. Am I Sports Med 1976; 4: 11-21.

11 Phelps D, Sonstegard DA, Mathews LS. Corticosteroid injection effects on the biomechanical properties of rabbit patellar tendons. Clin Orthop 1974; 100: 345-8.

12 Noyes FR, Nussbaum NS, Torvik PJ, Cooper S. Biomechanical and ultrastructural changes in ligaments and tendons after local corticosteroid injections. J Bone Joint Surg 1975; 57A: 876.

13 Goldie I. Local steroid therapy in painful orthopedic conditions. Scot Med J 1972; 17: 176-86.

14 Carstam N. The effect of cortisone on the formation of tendon adhesions and on tendon healing. Acta Chir Scand Suppl 1953; 85: 182.

15 Gonzalez RI. Experimental tendon repair within the flexor tunnels. J Bone Joint Surg 1953; 35A: 991-3.

16 Vogel HG. Zum Wirkung von hormonen auf physikalische und chemische eigenschaften des binde und stützgewebes. Arzneimittelforschung 1969; 19: 1981-96.

17 Wrenn RN, Goldner JL, Markee JL. An experimental study of the effect of cortisone on the healing process and tensile strength of tendons. J Bone Joint Surg 1954; 36A: 588-601.

18 Arner O, Lindholm A. Subcutaneous rupture of the Achilles tendon. Acta Chir Scand Suppl 1959; 239: 1-51.

19 Goldman S, Linscheid RL, Bickel WH. Disruptions of the tendo Achilles: analysis of 33 cases. Mayo Clin Proc 1969; 44: 28-35

20 Christensen I. Rupture of the Achilles tendon. Acta Chir Scan 1954; 106: 50-60.

21 Barfred T. Achilles tendon rupture. Acta Orthop Scand Suppl 1973; 152: 1-126.

22 Nillius SA, Nilsson BE, Westlin NE. The incidence of Achilles tendon rupture. Acta Orthop Scand 1976; 47: 118-21.

23 Nicholas JA. The Lower Extremity and Spine in Sports Medicine. Vol. 1. St. Louis: CV Mosby, 1986: 507.

24 Lea RB, Smith L. Non-surgical treatment of tendo Achilles rupture. J Bone Joint Surg 1972; 54-A: 1398-407.

25 Ljungqvist R. Subcutaneous partial rupture of the Achilles tendon. Acta Orthop Scand Suppl 1968; 113.

26 Williams JGP. Achilles tendon lesions in sport. Sports Med 1986; 3: 114-35.

27 Puddu G, Ippolito E, Postacchini F. A classification of Achilles tendon disease. Am J Sports Med 1976; 4: 145-50.

28 Williams JGP. Lesions of tendon attachments. Rheumatol Rehabil 1973; 12: 182-6.

29 Fox JM, Blazina ME, Jobe FW et al. Degeneration and rupture of the Achilles tendon. Clin Orthop 1975; 107: 221-4.

30 Ippolito E, Postacchini F, Puddu G. Le atteraziono structurali del tendine di Achillé. Medezin une Sport 1973; 26: 258.

31 Jacobs D, Martens M, van Audekercke R et al. Comparison of conservative and operative treatment of Achilles tendon rupture. Am J Sports Med 1978; 6: 107.

32 Clancy WG, Neidhart D, Brand RL. Achilles tendonitis in runners: a report of five cases. Am J Sports Med 1976; 4: 46-56.

33 Burry HC, Pool CJ. Central degeneration of the Achilles tendon. Rheumatol Rehabil 1973; 12: 177-81.

34 Lagergren C, Lindholm A. Vascular distribution in the Achilles tendon. Acta Chir Scand 1958/9; 111: 491-5.

35 Lee MLH. Bilateral rupture of Achilles tendon. Br Med J 1961; i: $1829-30$.

36 Rappaport G, Gerster JC. Les injections locales de corticostéroides en pratique rhumatologique. Médecine et Hygiène 1988; 46: 890-6.

37 d'Anglejan G, Dupuich C, Le Meignen P et al. Cortisone and tendon. Actualités Rhumatologiques 1983; 20: 293-7.

38 Bedi SS, Ellis W. Spontaneous rupture of the calcaneal tendon in rheumatoid arthritis after local steroid injection. Ann Rheum Dis 1970; 29: 494-5.

39 Bentley G, Goodfellow JW. Disorganization of the knees following intra-articular hydrocortisone injections. J Bone Joint Surg 1969; 51B: 498-502.

40 Cowan MA, Alexander S. Simultaneous bilateral rupture of the tendons due to triamcinolone. $\mathrm{Br}$ Med J 1961; i: 1658.

41 Ford LT, DeBender J. Tendon rupture after local steroid injection. South Med J 1979; 72: 827-30.

42 Karpmann RR, McComb JE, Volz RG. Tendon rupture following local steroid injection. Postgrad Med 1980; 68: 169-76.

43 Smaill GB. Bilateral rupture of Achilles tendons. $\mathrm{Br}$ Med J 1961; i: 1657. 
44 Pilgaard S. Akillesseneruptur under korticosteroidbehandling. Ugeskr Laeger 1962; 124: 1408-9.

45 Melmed EP. Spontaneous bilateral rupture of the calcaneal tendon during steroid therapy. J Bone Joint Surg 1965; 47B: $104-5$.

46 Chechick A, Amit Y, Israeli A, Horoszowski H. Recurrent rupture of the Achilles tendon induced by corticosteroid injection. Br J Sports Med 1982; 16: 89-90.

47 Kleinmann M, Gross AE. Achilles tendon rupture following steroid injection. J Bone Joint Surg 1983; 65A: 1345-7.

48 Halpern AA, Horowitz BG, Nagel DA. Tendon ruptures associated with corticosteroid therapy. West J Med 1977; 127: 378-82.
49 Skoech DU. Spontaneous partial subcutaneous ruptures of the tendo Achilles. Am J Sports Med 1981; 9: 20-2.

50 Denstad F, Roaas A. Surgical treatment of partial Achilles tendon rupture. Am J Sports Med 1979; 7: 15-17.

51 Shields CL. The Cybex II evaluation of surgically repaired Achilles tendon ruptures. Am J Sports Med 1978; 6: 369-72.

52 Hamilton WG. Foot and ankle injuries in dancers. Clin Sports Med 1988; 7: 155-8.

53 Renström P, Johnson RJ. Overuse injuries in sports. Sports Med 1985; 2: 316-33.

54 DaCruz DJ. Achilles paratendonitis: an evaluation of steroid injection. Br J Sports Med 1988; 22: 64-5. 